\title{
Opiate free anaesthesia and future of thoracic surgery anaesthesia
}

\author{
Chinmay Patvardhan, Mario Ferrante \\ Department of Anaesthesia, Royal Papworth Hospital, Cambridge, UK \\ Correspondence to: Chinmay Patvardhan. Royal Papworth Hospital, UK. Email: chinmay24@me.com.
}

Received: 11 December 2018; Accepted: 30 December 2018; Published: 24 December 2018.

doi: 10.21037 /jovs.2018.12.08

View this article at: http://dx.doi.org/10.21037/jovs.2018.12.08

\section{Introduction}

History of development of anaesthesia as a specialty is intricately linked with the use of opiates. The word Anaesthesia derives from the Greek word "ANAI $\Sigma \Theta H \Sigma I A$ " anaisthēsia, its meaning is: an 'without', aisthēsis 'sensation', but if we look back, we find that anaesthesia has older origins. It is well known that Sumerian in 4000 BCE already used Opium for pain relief. However, it was only in 1805 that pharmacist Friedrich Sertürner was able to isolate a new substance from opium, which he later named "morphium" after Morpheus, the God of dreams. The history of synthetic opioids begins after 1950, with the development of the 'modern' anaesthetic techniques. In 1962 the use of Fentanyl, the first synthetic opioid for use in anaesthesia, was described in Belgium. Since then the use of synthetic opiates in modern anaesthetic practice has expanded greatly and opiates have become the mainstay of providing effective analgesia after surgical procedures. High doses of opiates have been traditionally been used in cardiothoracic anaesthesia, which exploit the sedo-analgesic property of opiates combined with the relative cardio stability that follows from the high dosage of opiates.

However, opiates do not come without side effects. During the perioperative period, nausea and vomiting, dizziness, constipation, respiratory depression, puritus and delirium have a significant detrimental effect on recovery after surgery, especially among the elderly.

In the past decade, opiates have also been increasingly prescribed for management of chronic pain. Millions of these medicines are prescribed in western countries. This has resulted in an opiate prescription epidemic, which has resulted in over 200,000 people dying in the United States from Opiate Overdose between 1999-2016 (1).

There has been increasing awareness of problems associated with opiate overuse and misuse in the recent days. We know from medical literature that risk of opiate addiction is highest amongst people who have been on prescription opiate drugs. The risk of accidental overdose, death, addiction is higher among patients who have been prescribed opiates for a longer time. Perioperative prescription of opiates is often the first exposure of many of these patients to opiates which ultimately results in addiction (2).

As a result, there has been a re- emergence of multimodal analgesic strategies using pharmacology and the knowledge gained from better understanding of neuropathic pain and pain pathways (2). Drugs like Gabapentin and pregabalin, or antidepressants like duloxetine are being increasingly prescribed for their opiate sparing and analgesic effect during and after surgery in combination with the traditional non opioid drugs like paracetamol/ NSAIDS etc. Local anaesthetic medications like lignocaine is now being increasingly used as perioperative infusions with good effect. Clonidine and dexmedetomidine with their alpha agonistic effect on pain pathways have a relative opiate sparing effect. Anaesthetists are increasingly using these drugs in combination with nerve blocks, neuraxial blocks and wound infusion catheters in an attempt to reduce perioperative opiate use.

\section{Problems with opiate analgesia}

Opioid analgesia remains the corner stone of acute pain management in perioperative analgesic regimes. This is because of their high potency and efficacy. However as previously mentioned, they are not without their side effects. Opiate analgesia works through opioid receptors which are widely distributed through the body. Agonism at the $\mu$ receptors, which are widely distributed in the brain stem result in bradypnea, apnoea and reduction in minute 
ventilation. In perioperative setting, this effect is synergistic with benzodiazepines, and anaesthetic agents. This has a potential for causing serious respiratory depression leading to significant morbidity requiring prolonged mechanical ventilation and even mortality. This is particularly relevant in thoracic surgery, where an un intentional consequence of respiratory depression, from a well-intentioned prescription of perioperative opiates, can be disastrous.

This is because patients undergoing thoracic surgery/ lung resection, have a reduced functional residual capacity from the surgery and lung resection. They may have low FEV1, FVC or diffusion capacity and are prone to hypoxia. Any hypoventilation in perioperative period puts these patients at risk of developing hypercapnia, hypoxia and respiratory failure. This problem is also compounded by the narcotic and anti-tussive effect of opiates which depresses ciliary function causing sputum retention.

A more sinister effects of opiate analgesics which is not often appreciated is the immunosuppression and immunomodulation that occurs with administration of opiates (3). Natural killer (NK) cells are a subtype of innate Lymphocytes (also known as group I innate). These cells are responsible for eradication of tumour cells and virally infected cells in the body. They also secrete cytokines like interferon-gamma (IFN $\gamma$ ) and tumour necrosis factor $(\mathrm{TNF} \alpha)$ which modulate functions of other immune cells and inflammation, which aids in eradication of tumour cells and viral infections. Commonly used opiates such as morphine, codeine, fentanyl have all shown immunosuppressive properties in both pre-clinical and clinical studies. They cause suppression of NK cell function resulting in reduced ability of the NK cell to eradicate malignant cells. They also suppress the cytotoxic ability of NK cells. Morphine administration in clinical studies has been shown to have accelerate progression of cancer and increased susceptibility to infections. Studies specifically looking at lung cancer have suggested a plausible hypothesis that opiate administration directly increases lung cancer progression through the $\mathrm{Mu}$ - opiate receptor up regulation (4). These studies noted that there is a $5-10$-fold increase in $\mathrm{Mu}$ Opiate receptor expression on non-small cell lung cancer cell lines. they also noted that by knocking out the expression of the $\mathrm{Mu}$ receptor there was a significant decrease in invasiveness of the cancer and ability to metastasis (4).

\section{Regional anaesthesia and thoracic surgery}

Last decade has seen rapid increase in understanding how the neuro- inflammation and immune modulatory response of perioperative stress has an impact on oncological progression of cancers. We know that an effective inhibition of perioperative stress response has a beneficial effect on outcomes after major surgery. Regional anaesthesia i.e., neuraxial blocks (spinal, epidural or paravertebral) are effective in reducing the perioperative stress response. They also have beneficial effects in reducing blood loss, transfusion requirements, and provide superior analgesia. Therefore, it is not surprising that regional analgesia has been used extensively in thoracic surgery. As we begin to understand the effects of neuro-inflammation and immune modulation, evidence is beginning to emerge that the use of regional anaesthesia may have superior analgesic effects and may even have survival benefit in certain types of cancers (e.g., breast cancer). Local anaesthetic agents like lignocaine have also been shown to have anti-tumour activity. Regional anaesthetic techniques are popular in enhanced recovery programs as they allow for reduction in opiate requirement and general anaesthesia during surgery. Traditionally, thoracic epidural anaesthesia (TEA) has been the gold standard for provision of perioperative analgesia after thoracic surgery. This is because of its superior analgesia, which allows for the patient to cough, mobilise and rehabilitate effectively after a major thoracotomy. However, this is not without its problems. TEA can lead to incidents of significant hypotension, urinary retention and inadvertent administration of fluid, which can be detrimental to lung surgery patients. UK pneumonectomy outcome study (UKPOS) published in 2009 showed epidural analgesia to be an independent risk factor for development of major complication after pneumonectomy. They also paradoxically increase the length of stay by the fact that the patient remains connected to the epidural pump and therefore remain in the hospital. There is always the added serious risk of associated with insertion of an epidural catheter, namely, nerve damage and epidural hematoma. However, as epidurals have fallen out of favour in the U.K., paravertebral blocks combined with peripheral nerve blocks can provide equally effective analgesia. The newer truncal nerve blocks like PECS I \&II, serratus plane block is increasingly being used in thoracic surgery with good effect. The popular appeal of these blocks is that it allows for the patient to be mobilised without being connected to a pump, and promotes faster rehabilitation, mobilisation and discharge from hospital.

The other factor that has promoted the popularity of nerve blocks in thoracic surgery is the increasing use of 
smaller incisions and newer techniques like sub xiphoid approach, uniporter video-assisted approach. These minimally invasive techniques described elsewhere in this book have revolutionised the perioperative conduct of thoracic anaesthesia. Smaller incisions mean lesser appetite among surgeons and anaesthetists for being 'heavy handed' with central neuraxial blocks.

This complex interplay of various push and pull factors, on top of increasing awareness of unintentional side effects of opiate analgesics in onco- surgery has led to a gradual evolution of anaesthetic techniques. In the forefront of this is Opioid Free Anaesthesia (OFA).

\section{Opioid free anaesthesia and future of thoracic anaesthesia}

Anaesthesia is a trial of hypnosis, analgesia and provision of muscle relaxation. Traditionally analgesia has always been used with strong synthetic opioid medications like fentanyl, alfentanil or remifentanil or natural opiates like morphine and its derivatives. Opioid free anaesthesia is a unique anaesthetic technique, which avoid all use of opioid medications during anaesthesia but instead utilises a combination alternative medication like $\alpha-1$ agonists (i.e., clonidine or dexmedetomidine), local anaesthetic infusions (e.g., lignocaine), NMDA antagonists (e.g., ketamine, magnesium) to provide the analgesia and sympatholysis, while maintaining the quality of perioperative amnesia, hypnosis and muscle relaxation during surgery. The innovative idea behind this is that by avoiding opiates, this technique without compromising on the quality of anaesthesia, enhances the quality of recovery after anaesthesia by avoiding all the noxious side effects associated with opiates (5).

Another added benefit of using a combination of adjunctive medication is that it blocks the acute pain pathways at multiple levels, and in theory may beneficial in reducing chronic pain and hyperalgesia which is commonly associated with thoracic surgery.

Published evidence suggests OFA has been successfully used bariatric surgery, colorectal surgery, cholecystectomies, orthopaedic surgery and the experience of anaesthetists is rapidly increasing. However at present, there are currently no published papers trialling OFA in thoracic surgery.

Because of the unique anatomy of the lung and innervation of the chest wall, thoracic surgery pain lends itself well to nerve blockade with paravertebral or peripheral nerve plexus blocks (i.e., pectoral muscle plane block, serratus anterior plane block or intercostal nerve block). The nociceptive visceral aspect of the pain conducted through the Phrenic or Vagus nerve can be blocked during surgery with local anaesthetic without any detriment to respiratory function thereby reducing the 'referred' aspect of post thoracotomy pain.

Combining these 'nerve-blocks' along with an opioid free anaesthetic has the potential to significantly improve the quality of patient recovery and overall experience after minimally invasive thoracic surgery. Using an opioid free technique has the potential to virtually eliminate the noxious side effects of opioid medications, while continuing to provide high quality analgesia to patient. This allows for faster recovery from anaesthesia and shortening rehabilitation time. There is also a potential economic benefit - by reducing morbidity associated with opioid medication (i.e., respiratory depression, delirium, constipation, narcosis) OFA can reduce the length of stay in lung surgery patients. This technique can also virtually eliminate all the undesirable immunological and potential onco-proliferative side effects associate with perioperative opiate administration in lung cancer surgery.

However all this would have to be proven by a well conducted prospective randomised trial. But if the results from non-cardiac surgery are anything to go by, then we should be encouraged to trial OFA in thoracic surgery.

In our institution, we are currently trialling OFA for thoracic surgery in a safe and scientific fashion. Our initial results are encouraging, however we have not reached a critical mass to submit a peer review publication at the timing of writing this chapter. Our early informal experience at this stage suggests that the overall analgesia and patient experience is non-inferior to standard anaesthetic technique used in our institution. However, these initial results have not been scrutinised or published in a peer review journal yet.

\section{Conclusions}

The practice of opioid free anaesthesia shows the potential to innovate and transform the practice of thoracic anaesthesia and enhanced recovery programs after minimally invasive thoracic surgery. Although there are no proven clinical trials at this stage, our initial experience is promising. Future research would ultimately prove if the technique works and reveal the unknown-unknowns. 


\section{Acknowledgments}

Funding: None.

\section{Footnote}

Provenance and Peer Review: This article was commissioned by the editorial office, fournal of Visualized Surgery. The article did not undergo external peer review.

Conflicts of Interest: Both authors have completed the ICMJE uniform disclosure form (available at http://dx.doi. org/10.21037/jovs.2018.12.08). The authors have no conflicts of interest to declare.

Ethical Statement: The authors are accountable for all aspects of the work in ensuring that questions related to the accuracy or integrity of any part of the work are appropriately investigated and resolved.

Open Access Statement: This is an Open Access article distributed in accordance with the Creative Commons Attribution-NonCommercial-NoDerivs 4.0 International License (CC BY-NC-ND 4.0), which permits the non-

doi: 10.21037/jovs.2018.12.08

Cite this article as: Patvardhan C, Ferrante M. Opiate free anaesthesia and future of thoracic surgery anaesthesia. J Vis Surg 2018;4:253. commercial replication and distribution of the article with the strict proviso that no changes or edits are made and the original work is properly cited (including links to both the formal publication through the relevant DOI and the license). See: https://creativecommons.org/licenses/by-nc-nd/4.0/.

\section{References}

1. Seth P, Rudd RA, Noonan RK, et al. Quantifying the Epidemic of Prescription Opioid Overdose Deaths. Am J Public Health 2018;108:500-2.

2. Kumar K, Kirksey MA, Duong S, et al. A review of opioidsparing modalities in perioperative pain management: methods to decrease opioid use postoperatively. Anesth Analg 2017;125:1749-60.

3. Kaye AD, Patel N, Bueno FR, et al. Effect of opiates, anesthetic techniques, and other perioperative factors on surgical cancer patients. Ochsner J 2014;14:216-28.

4. Mathew B, Lennon FE, Siegler J, et al. The novel role of the mu opioid receptor in lung cancer progression: a laboratory investigation. Anesth Analg 2011;112:558-67.

5. Mulier JP. Opioid free anaesthesia (OFA) a Paradigm shift. Available online: https://www.researchgate.net/ publication/278307444_Opioid_free_Anaesthesia 